\title{
Can We Use the Posterior Condylar Offset as a Predictive Factor for Overhang of the Implant in Total Knee Arthroplasty?
}

\author{
Yoon-Seok Youm, MD, Sung-Do Cho, MD, Jin Eo, MD, Ki-Bong Park, MD and Sun-Ho Lee, MD \\ Department of Orthopedic Surgery, Ulsan University Hospital, University of Ulsan College of Medicine, Ulsan, Korea
}

Purpose: We tried to demonstrate whether the posterior condylar offset (PCO, the distance from the femoral diaphysis posterior cortex to the posterior condylar margin) and ratio (PCOR, dividing PCO by the maximum antero-posterior diameter of the distal femur) could be used as predictive factors for overhang of the implants or using gender implants in total knee arthroplasty (TKA).

Materials and Methods: One hundred and one women who underwent TKA using NexGen ${ }^{\circledR}$ (LPS) implants, were analyzed prospectively. After distal femoral resection, the mediolateral (ML) width was measured at four points (anterior, distal anterior, distal posterior and posterior) and compared with the ML width of the implant. The aspect ratio (AR, ML/AP ratio) and anterior/distal posterior ML width (Ant/DP) were calculated. Preoperative radiographic PCO and PCOR were measured. Differences of PCO, PCOR, AR and Ant/DP according to the size were analyzed and correlations between PCO, PCOR and AR were also analyzed. The patients were classified into two groups according to the presence of overhang, and differences of each parameter were compared between the two groups.

Results: The size of the implant was positively correlated PCO, not significantly correlated with PCOR, and negatively correlated with AR and Ant/ DP. PCO and PCOR and AR showed no correlation with each other. PCO and PCOR were not significantly different between the two groups. However, AR and Ant/DP were statistically low in the group with overhang.

Conclusions: Preoperative radiographic PCO or PCOR could not be used as a predictive factor for overhang of the implants or using gender implants in TKA.

Key words: Total knee arthroplasty, Distal femur, Posterior condylar offset, Aspect ratio, Overhang.

\section{Introduction}

With better understanding of the differences between genders in the anatomy of the distal femur and the proximal tibia, the use of gender-specific implants in total knee arthroplasty (TKA)

Received April 7, 2011; Revised (1st) May 3, 2011; (2nd) June 30, 2011; Accepted July 10, 2011.

Correspondence to: Sung-Do Cho, MD.

Department of Orthopedic Surgery, Ulsan University Hospital, University of Ulsan College of Medicine, 290-3 Jeonha-dong, Dong-gu, Ulsan 682-714, Korea

Tel: +82-52-250-7129, Fax: +82-52-235-2823

Email: sdcho@uuh.ulsan.kr

This is an Open Access article distributed under the terms of the Creative Commons Attribution Non-Commercial License (http://creativecommons.org/licenses/by-nc/3.0/) which permits unrestricted non-commercial use, distribution, and reproduction in any medium, provided the original work is properly cited.

Copyright @ 2011. THE KOREAN KNEE SOCIETY

www.jksrr.org has been addressed in many studies. Gender-specific femoral implants are shaped narrower mediolaterally than the traditional ones to better fit the anatomy of the female knee joint, thus reducing the risk of overhang. The presence of overhang can be identified intraoperatively by measuring the mediolateral $(\mathrm{ML})$ width and the anteroposterior (AP) diameter of the distal femur and has been known to be associated with the aspect ratio (AR, ML/AP ratio) $)^{1,2)}$.

Posterior condylar offset (PCO) has been used as a term to describe the distance from the femoral diaphysis posterior cortex to the posterior condylar margin and the concept of posterior condylar offset ratio (PCOR), the ratio of PCO to the maximum AP diameter of the distal femur, has been suggested ${ }^{3,4)}$. PCO and PCOR can be measured with ease on plain lateral radiographs before surgery. Therefore, we thought if an association with overhang was observed, PCO and PCOR would be useful predictive factors for overhang before surgery. In this study, we investigated whether the PCO or PCOR could be used as predictive factors for overhang or the use of a gender-specific 
implant. Our hypothesis was that the PCO and PCOR are positively correlated with the AP diameter and are negatively correlated with the AR.

\section{Materials and Methods}

A total of 101 female patients (147 cases) who underwent total knee arthroplasty (TKA) using Nexgen PS implants (Nexgen ${ }^{\circledR}$ LPS, Zimmer, Warsaw, IN, USA) between March 2008 and February 2010 were included in this prospective study. Their mean age was 69.8 years (range, 59 to 86 years). Bilateral TKA was performed in 35 patients. Distal femoral resection was performed using a measured resection technique in all

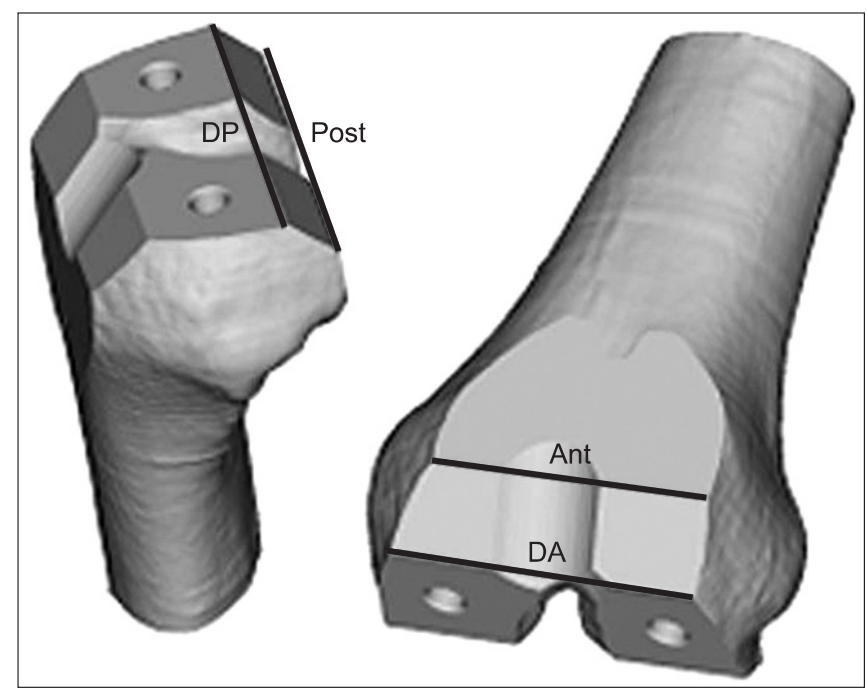

Fig. 1. Measurements of the distal femur at 4 points -anterior (Ant), distal anterior (DA), distal posterior (DP) and posterior (Post). cases. The femur was cut first and the femoral component size was determined according to the amount of resection of the posterior femoral condyle based on the AP diameter of the distal femur. The size of the component was $\mathrm{C}, \mathrm{D}$, and $\mathrm{E}$ in 27 (18.4\%), 75 (51.0\%), and 45 (30.6\%) cases, respectively. After the distal femoral resection, the mediolateral (ML) width of the femur was measured at 4 points (anterior, distal anterior, distal posterior, and posterior) (Fig. 1). The distal femoral osteophytes were removed to reduce measurement errors. The ML width of the femoral component measured at the same 4 points was identified in the supplier catalog (Fig. 2). When the ML width of a femoral component was larger than that of the femur at least at one of the 4 points, it was considered overhang and a gender-

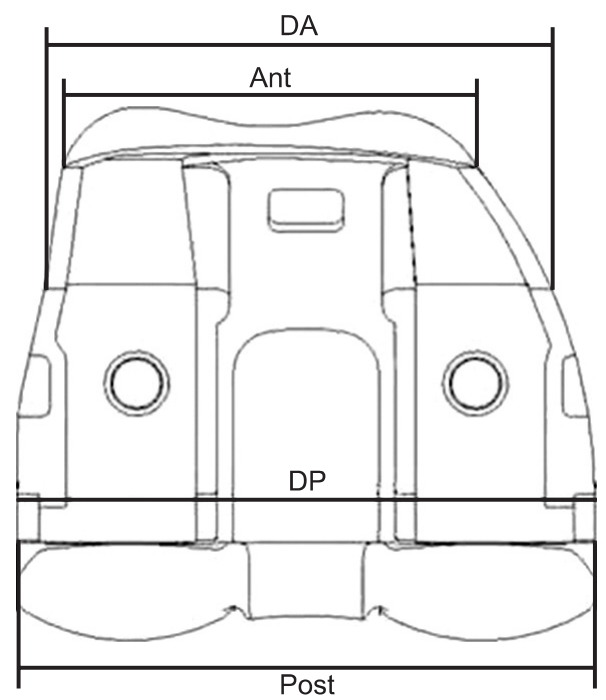

Fig. 2. Measurements of the femoral implant at 4 points - anterior (Ant), distal anterior (DA), distal posterior (DP) and posterior (Post).
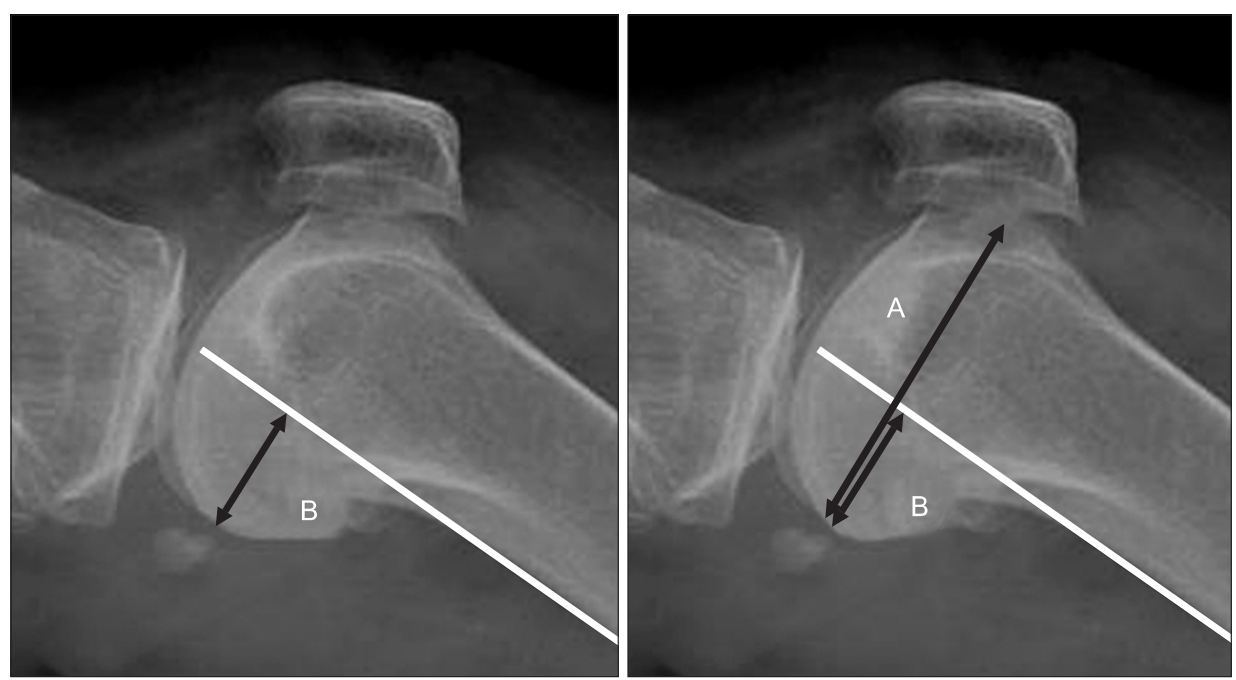

Fig. 3. Posterior condylar offset (B) and posterior condylar offset ratio $(B / A)$ were demonstrated on the preoperative lateral radiograph. 
specific implant (Nexgen ${ }^{\circledR}$ LPS-flex Gender, Zimmer, Warsaw, IN, USA) was used, if necessary. AR (ML/AP ratio) and the ratio of the anterior ML width to the distal posterior ML width (Ant/ DP) were calculated according to the implant size. PCOR was obtained by dividing the PCO by the maximum AP diameter of the distal femur that were measured on plain lateral radiographs (Fig. 3). The differences in PCO, PCOR, AR, and Ant/DP according to the implant size were analyzed and the relationships between PCO and PCOR and AR were assessed using the Pearson's correlation coefficient analysis. Overhang was observed in 11 cases ( 2 with size C, 2 with size D, and 7 with size E), not in the remaining 136 cases. Of the 11 overhang cases, a genderspecific implant was used in 7 cases except for the 4 minor cases. Differences between the 11 overhang cases (group I) and the remaining 136 cases (group II) in terms of PCO, PCOR, AR, and Ant/DP were statistically analyzed using the Mann-Whitney $U$ test. Statistical significance was set at $\mathrm{p}<0.05$.

\section{Results}

The preoperative PCO of the distal femur was correlated positively with the implant size, but PCOR was not related with the implant size. AR was negatively correlated with the implant size at all of the 4 points of the distal femur, especially at the anterior (Table 1). Ant/DP was negatively correlated with the implant size (Table 1). No significant correlation was found between PCO and AR and between PCOR and AR not only in all of the cases but also in each group divided according to the size of femoral component. The preoperative PCO and PCOR were not statistically different between the group I (overhang) and group II (non-overhang), but AR and Ant/DP in group I were significantly less than those in group II (Table 2).

\section{Discussion}

The anatomical differences between males and females

Table 1. Differences of PCO, PCOR, AR, and Ant/DP According to the Femoral Component Size

\begin{tabular}{lrrrr}
\hline \multicolumn{1}{c}{ Size } & \multicolumn{1}{c}{ C } & \multicolumn{1}{c}{ D } & \multicolumn{1}{c}{ E } & p-value \\
\hline PCO $(\mathrm{mm})$ & $26.78 \pm 2.84$ & $29.43 \pm 3.49$ & $30.92 \pm 3.28$ & $<0.001$ \\
PCOR & $0.44 \pm 0.06$ & $0.46 \pm 0.05$ & $0.46 \pm 0.05$ & 0.186 \\
AR & $1.19 \pm 0.05$ & $1.15 \pm 0.04$ & $1.11 \pm 0.03$ & $<0.001$ \\
Ant/DP & $0.74 \pm 0.06$ & $0.72 \pm 0.04$ & $0.70 \pm 0.04$ & 0.002 \\
\hline
\end{tabular}

PCO: posterior condylar offset, PCOR: posterior condylar offset ratio, AR: aspect ratio, Ant/DP: anterior medio-lateral width/distal posterior medio-lateral width. including the lower limb alignment and the distal femur anatomy have been the subjects of many studies. Chin et al. ${ }^{1)}$ and Hitt et al. ${ }^{2)}$ reported that the ML width of the distal femur is generally narrower in women compared to men when the AP diameter is same. Booth ${ }^{5)}$ suggested the need to develop new implants designed to better allow for anatomic variations. Such variations exist between genders ${ }^{3,5)}$ and are more pronounced among the races $^{6-10)}$.

Conley et al. ${ }^{11)}$ suggested the need for a gender-specific implant design based on their findings that there are anatomic differences between male and female knees regarding the $\mathrm{Q}$ angle, prominence of the anterior medial and anterior lateral femoral condyles, and the aspect ratio. However, Merchant et $\mathrm{al}^{12)}$ reported that no gender differences were noted in the $\mathrm{Q}$ angle and the prominence of the anterior medial and anterior lateral femoral condyles and the difference in the aspect ratio was not clinically significant. In addition, their analysis of the literature showed that women had better results than men after TKA using traditional implants contrary to their expectation that the use of implants that do not take anatomical differences into account would produce worse results in women. MacDonald et al. ${ }^{13)}$ compared the clinical results and reoperation rates between males and females using the Western Ontario and McMaster Universities Osteoarthritis Index score, Short-form 12, and Knee Society clinical ratings score and found that women had better clinical results and low reoperation rates overall. In this study, we did not use gender-specific implants in most of the cases (136 of 147 cases, 92.5\%) because overhang was not observed in them.

Medial or lateral overhang causes soft tissue irritation and influences the joint stability. The use of an implant smaller than the appropriate size leaves the cancellous bone exposed, which could lead to an increase in postoperative bleeding and osteolysis by polyethylene wear particle in the long-term. An overhang

Table 2. Differences of PCO, PCOR, AR, and Ant/DP between Group I and Group II

\begin{tabular}{lrrr}
\hline & \multicolumn{1}{c}{ Group I $^{\mathrm{a})}$} & Group II $^{\mathrm{b})}$ & p-value \\
\hline PCO $(\mathrm{mm})$ & $29.20 \pm 5.14$ & $29.42 \pm 3.46$ & 0.805 \\
PCOR & $0.46 \pm 0.07$ & $0.46 \pm 0.05$ & 0.735 \\
AR & $1.08 \pm 0.03$ & $1.15 \pm 0.05$ & $<0.001$ \\
Ant/DP & $0.68 \pm 0.05$ & $0.72 \pm 0.05$ & 0.032 \\
\hline
\end{tabular}

PCO: posterior condylar offset, PCOR: posterior condylar offset ratio, AR: aspect ratio, Ant/DP: anterior medio-lateral width/distal posterior medio-lateral width.

${ }^{a)}$ Group I: group of cases with overhang of the femoral implant.

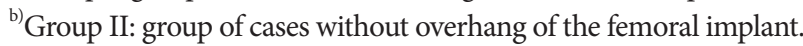


caused by a larger-sized implant can result in changes in the soft tissue tension and in the patellofemoral contact pressure.

Bellemans et al. ${ }^{3)}$ reported that PCO was positively correlated with the flexion angle in the cruciate-retaining TKA and PCOR appeared to influence the postoperative flexion angle ${ }^{4)}$. Considering that PCO and PCOR are closely related to the AP diameter of the femur, we expected that PCO and PCOR that can be easily measured on preoperative radiographs would be positively correlated with the AP diameter of the femur and negatively correlated with AR (ML/AP). However, we could not find a significant correlation between them. We attributed this to the fact that the ML width had no association with PCO although the AP diameter is associated with PCO. In a knee with a small $\mathrm{AR}$, the risk of overhang increases when an implant that has the same AP diameter of the femur is used because the ML width of the implant could be larger than that of the femur. Indeed, AR was significantly small in group I (cases with overhang) in our study. Ant/DP was remarkably small in group I and we attributed this to the drastic decrease in the AR at anterior, although the AR decreased at all of the 4 points. AR and Ant/DP appeared to negatively correlate with the implant size. Although significance was difficult to find due to the small number of overhang cases, we thought a correlation could be observed with large implants. $\mathrm{AR}$ and Ant/DP were significantly different between group I and group II, suggesting a correlation with overhang. Unfortunately, $\mathrm{AR}$ and Ant/DP have a limitation as a predictive factor because they can only be measured intraoperatively after distal femoral resection.

The limitation of this study is that the number of overhang cases was small, 11 (7.5\%) out of 147 cases. Further studies including larger number of overhang cases would be necessary.

\section{Conclusions}

PCO and PCOR that can be measured on preoperative radiographs could not be used as predictive factors for implant overhang in TKA.

\section{References}

1. Chin KR, Dalury DF, Zurakowski D, Scott RD. Intraoperative measurements of male and female distal femurs during primary total knee arthroplasty. J Knee Surg. 2002;15:213-7.

2. Hitt K, Shurman JR 2nd, Greene K, McCarthy J, Moskal J,
Hoeman T, Mont MA. Anthropometric measurements of the human knee: correlation to the sizing of current knee arthroplasty systems. J Bone Joint Surg Am. 2003;85(Suppl 4):115-22.

3. Bellemans J, Banks S, Victor J, Vandenneucker H, Moemans A. Fluoroscopic analysis of the kinematics of deep flexion in total knee arthroplasty. Influence of posterior condylar offset. J Bone Joint Surg Br. 2002;84:50-3.

4. Soda Y, Oishi J, Nakasa T, Nishikawa K, Ochi M. New parameter of flexion after posterior stabilized total knee arthroplasty: posterior condylar offset ratio on X-ray photographs. Arch Orthop Trauma Surg. 2007;127:167-70.

5. Booth RE Jr. Sex and the total knee: gender-sensitive designs. Orthopedics. 2006;29: 836-8.

6. Ho WP, Cheng CK, Liau JJ. Morphometrical measurements of resected surface of femurs in Chinese knees: correlation to the sizing of current femoral implants. Knee. 2006;13:12-4.

7. Kwak DS, Surendran S, Pengatteeri YH, Park SE, Choi KN, Gopinathan P, Han SH, Han CW. Morphometry of the proximal tibia to design the tibial component of total knee arthroplasty for the Korean population. Knee. 2007;14:295300.

8. Shelbourne KD, Gray T, Benner RW. Intercondylar notch width measurement differences between African American and white men and women with intact anterior cruciate ligament knees. Am J Sports Med. 2007;35:1304-7.

9. Uehara K, Kadoya Y, Kobayashi A, Ohashi H, Yamano Y. Anthropometry of the proximal tibia to design a total knee prosthesis for the Japanese population. J Arthroplasty. 2002;17:1028-32.

10. Vaidya SV, Ranawat CS, Aroojis A, Laud NS. Anthropometric measurements to design total knee prostheses for the Indian population. J Arthroplasty. 2000;15:79-85.

11. Conley S, Rosenberg A, Crowninshield R. The female knee: anatomic variations. J Am Acad Orthop Surg. 2007;15(Suppl 1):S31-6.

12. Merchant AC, Arendt EA, Dye SF, Fredericson M, Grelsamer RP, Leadbetter WB, Post WR, Teitge RA. The female knee: anatomic variations and the female-specific total knee design. Clin Orthop Relat Res. 2008;466:3059-65.

13. MacDonald SJ, Charron KD, Bourne RB, Naudie DD, McCalden RW, Rorabeck CH. The John Insall Award: gender-specific total knee replacement: prospectively collected clinical outcomes. Clin Orthop Relat Res. 2008;466: 2612-6. 